\title{
Spatial Poincaré Plots as Descriptors of Speckle Pattern Second-Order Statistics
}

\author{
Anindya Majumdar, and Sean J. Kirkpatrick* \\ Department of Biomedical Engineering, Michigan Technological University, Houghton, MI 49931, USA \\ * e-mail: sjkirkpa@mtu.edu
}

\begin{abstract}
It is demonstrated herein that the use of spatial Poincaré plots provides an efficient means to describe short and long-range correlations in the spatial structure of the measured intensity distribution of scattered coherent fields. The intensity distribution over a row of pixels in single frames of speckle fields with varying speckle sizes was considered. Statistical descriptors from the spatial Poincaré plots for these intensity data with variable lags were used to estimate the short and long-term variations in the measured intensities, and from these descriptors, the minimum speckle size in the speckle patterns was estimated. This approach yielded similar results for speckle size estimates as the more standard method of calculating the power spectral density of the intensity pattern and simultaneously provided information on the relative contributions of short-term and long-term variations in the measured intensity to the spatial structure of the scattered fields. (C) 2017 Journal of Biomedical Photonics \& Engineering.
\end{abstract}

Keywords: coherence optics; statistical optics; speckle; Fourier optics; signal processing; speckle imaging.

Paper \#3231 received 12 Jul 2017; revised manuscript received 19 Sep 2017; accepted for publication 19 Sep 2017; published online 30 Sep 2017. doi: 10.18287/JBPE17.03.030501.

\section{References}

1. J. C. Dainty (Ed.), Laser Speckle and Related Phenomena, 2nd edition, Springer Verlag (1984).

2. X. Zhao, and G. Zhao, "Surface roughness measurement using spatial-average analysis of objective speckle pattern in specular direction," Optics and Lasers in Engineering 47(11), 1307-1316 (2009).

3. R. Jones, and C. Wykes, Holographic and Speckle Metrology, 2nd edition, Cambridge University Press (1989).

4. A. F. Fercher, and J. D. Briers, "Flow visualization by means of single-exposure speckle photography," Optics Communications 37(5), 326-330 (1981).

5. Y. Aizu, and T. Asakura, "Coherent optical techniques for diagnostics of retinal blood flow," Journal of Biomedical Optics 4(1), 61-75 (1999).

6. P. Li, S. Ni, L. Zhang, S. Zeng, and Q. Luo, "Imaging cerebral blood flow through the intact rat skull with temporal laser speckle imaging," Optics Letters 31(12), 1824-1826 (2006).

7. J. C. Ramirez-San-Juan, R. Ramos-Garcia, I. Guizar-Iturbide, G. Martinez-Niconoff, and B. Choi, "Impact of velocity distribution assumptions on simplified laser speckle imaging equation," Optics Express 16(5), 31973203 (2008).

8. K. Khaksari, and S. J. Kirkpatrick, "Laser speckle contrast imaging is sensitive to advective flux," Journal of Biomedical Optics 21(7), 076001 (2016).

9. J. W. Goodman (Ed.), Speckle Phenomena in Optics: Theory and Applications, Roberts \& Company, Englewood, CO (2007). ISBN 0-9747077-9-1.

10. S. J. Kirkpatrick, D. D. Duncan, and E. M. Wells-Gray, "Detrimental effects of speckle-pixel matching in laser speckle contrast imaging," Optics Letters 33(24), 2886-2888 (2008).

11. K. Khaksari, and S. J. Kirkpatrick, "Combined effects of scattering and absorption on laser speckle contrast imaging," Journal of Biomedical Optics 21(7), 076002 (2016). 
12. M. Brennan, M. Palaniswami, and P. Kamen, "Distortion properties of the interval spectrum of IPFM generated heartbeats for heart rate variability analysis," IEEE Transactions on Biomedical Engineering 48(11), 1251-1264 (2001).

13. D. D. Duncan, and S. J. Kirkpatrick, "The copula a tool for simulating speckle dynamics," Journal of the Optical Society of America A 25(1), 231-237 (2008).

Laser speckle patterns are obtained as a result of the random interference of coherent light scattering from a rough surface or a scattering volume [1]. Speckle patterns have been used extensively for a variety of metrology applications including surface roughness [2], strain measurement [3] and fluid flow [4-8]. The second-order statistics of a speckle pattern provide a description of the spatial structure of the pattern, with the minimum size of a speckle, or the minimum correlation length of the pattern, being an important parameter in the characterization of this structure [9]. It has been noted in the literature that proper spatial sampling of the intensity of the scattered field is an important experimental consideration in speckle-based metrology applications [10]. In order to meet the spatial Nyquist criteria, the minimum speckle size of the measured intensity pattern should be at least two pixels [8]. Goodman [9] introduced the $M$ parameter which can be interpreted as the number of speckles per pixel and may be used as a means of quantifying this. In practice, the minimum speckle size has traditionally been estimated in two dimensions in terms of the width of the power spectral density (PSD) function of the measured intensity distribution [9]

$$
P S D=|\Im\{I(x, y)\}|^{2},
$$

where $\mathfrak{I}$ represents the Fourier Transform operator and $I(x, y)$ is the distribution of the measured intensity of the speckle field. The typical plot of the PSD function is shown in Fig. 1. The minimum speckle size, $\Lambda$, is estimated from this distribution as $[8,11]$

$$
\Lambda=\frac{2 D}{W_{P S D}},
$$

where $D$ is the width of the speckle image and $W_{P S D}$ is the width of the PSD function [9]. Note that the PSD is simply the Fourier transform of the autocorrelation function and as such is a measure of the correlation length in the speckle pattern.

A Poincaré plot is a statistical tool to study variations, or, alternatively, self-similarity in a quantity. In these plots, sequential measures of the quantity of interest are plotted against the previous measures. Thus, the $i^{\text {th }}$ measure is plotted against the $(i+1)^{t h}$ measure, for $\mathrm{i}=1,2 \ldots N-1$, where $N$ is the total number of measurements available in the data. In biomedical signal processing, these plots have been used extensively to study heart rate variability over time [12]. Specific standard descriptors $S D 1$ and $S D 2$ can be obtained from the data. These descriptors are mathematically defined as [12]

$$
\begin{aligned}
& S D 1=\operatorname{Var}\left(\frac{1}{\sqrt{2}} X_{n}-\frac{1}{\sqrt{2}} X_{n+1}\right)^{\frac{1}{2}}=\frac{1}{\sqrt{2}}\left\{\operatorname{Var}\left(X_{n}-X_{n+1}\right)\right\}^{\frac{1}{2}} \\
& S D 2=\left\{2 \operatorname{Var}(X)-S D 1^{2}\right\}^{\frac{1}{2}},
\end{aligned}
$$

where $X_{n+1}$ and $X_{n}$ represent the current and previous measures, respectively and $\operatorname{Var}(X)$ stands for the variance in the measurement of $X$. As can be seen from Eq. (3), $S D 1$ is a measure of the standard deviation of the differences in successive data points. This is commonly known as the standard deviation in successive differences in statistics [12]. Thus, the descriptor $S D 1$ represents the short-term variations in the data [12]. Also, from the expression for $S D 2$, it is noted that this represents the difference between the overall variations in the data, $\operatorname{Var}(X)$, and the variations attributed to short-term differences, SD1. Thus, the descriptor SD2 effectively represents the long-term variations in the data. By these definitions, the ratio $S D 2 / S D 1$ is an indication of the prominence of longterm variations in the data, compared to short-term variations [12]. We have referred to this ratio as the ellipticity $e$ of the data. We have chosen this nomenclature, as the descriptors $S D 2$ and $S D 1$ are the semi-major and semi-minor axes, respectively, of an ellipse commonly used to study information from these plots [12] (Fig. 2).

As demonstrated by Brennan, Palaniswami and Kamen [12], for uncorrelated data, this ratio is unity. The larger the deviation of this ratio is from unity, the greater the correlation among the data points. In our research, we have made use of this ability of the Poincaré plot descriptors to indicate whether or not the intensity in neighboring pixels is correlated, which ultimately reflects the minimum speckle size in the pattern.

In order to take advantage of the ability or Poincaré plots to demonstrate correlation between neighboring data points, the traditional definition of Poincaré plot descriptors were modified for the purposes of this study. Instead of plotting every $(i+1)^{t h}$ measurement against the $i^{\text {th }}$ measurement, we introduced a coarsing factor $k$, such that every $(i+k)^{t h}$ measurement was plotted against the $i^{t h}$ measurement, for $i=1,2, \ldots N-k$, with $N$ being the number of data points (pixels) available. In previous publications [12], similar descriptors have been referred to as lag $m$ Poincaré descriptors. Thus, similar to Eq. 2, the modified descriptors are defined as 


$$
\begin{aligned}
& S D 1_{k}=\operatorname{Var}\left(\frac{1}{\sqrt{2}} X_{n}-\frac{1}{\sqrt{2}} X_{n+k}\right)^{\frac{1}{2}} ; \\
& S D 2_{k}=\left\{2 \operatorname{Var}(X)-S D 1_{k}^{2}\right\}^{\frac{1}{2}} .
\end{aligned}
$$

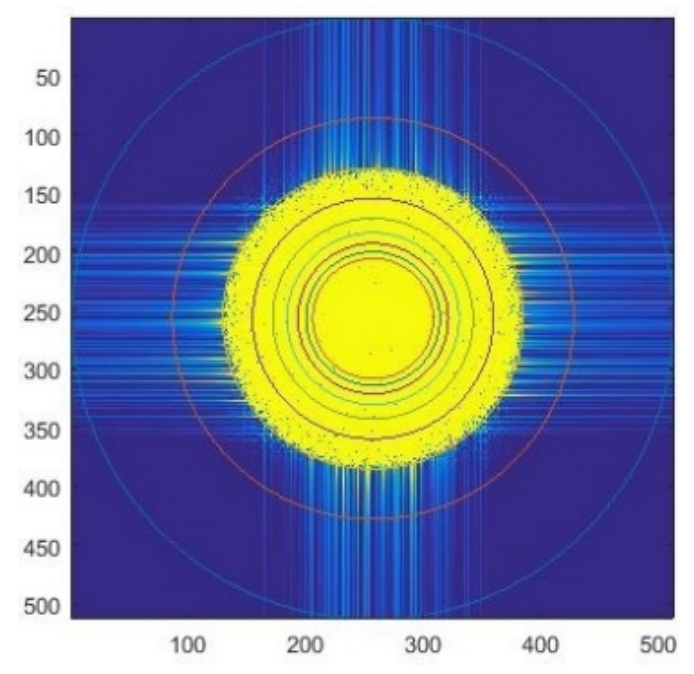

Fig. 1 The Power Spectral Density (PSD) of a frame of $512 \times 512$ pixels in a speckle field. It can be seen that the PSD width is half the overall width of the field, which in accordance with (1) gives a speckle size of 4 pixels. For easy visualization, concentric circles corresponding to each minimum speckle size from 2 to 10 pixels is drawn on the PSD. The outermost circle corresponds to size $=2$ pixels and each subsequent circle inwards corresponds to an increase in size by 1 pixel.

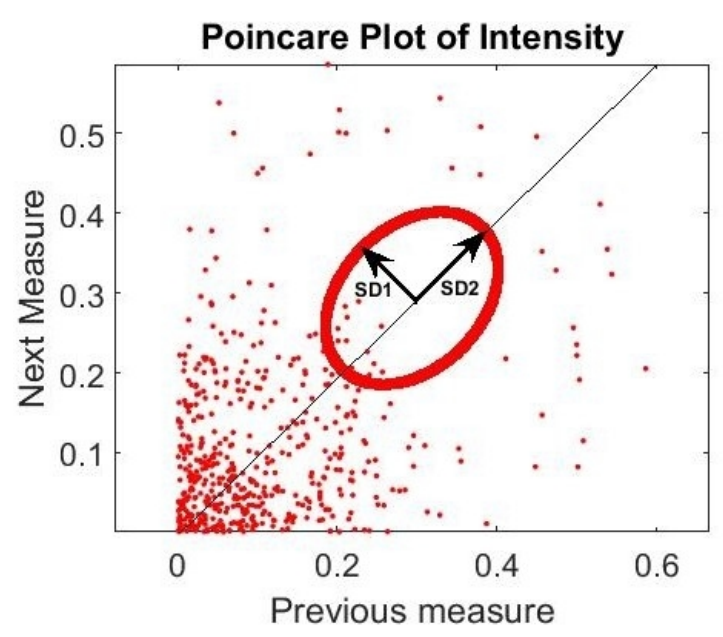

Fig. 2 A typical Poincaré plot. Any particular point represents a data point plotted in vertical axis against the previous data point in the horizontal axis.

Speckle fields were generated using an algorithm as described by Duncan and Kirkpatrick [13]. Using this algorithm, we generated speckle patterns with known minimum speckle sizes varying between 2 and 9 pixels. The intensity distribution of individual rows in these speckle patterns was examined. It has been shown previously [8] that the intensity PSD width accurately gives the minimum speckle size input by the user while implementing this algorithm. Our objective herein was to use modified spatial Poincare plot descriptors of the intensity distribution along a slice (single row) in the speckle pattern to indicate correlation lengths along this row of speckles and ultimately describe the spatial structure (i.e, the minimum speckle size) of the speckle pattern.

The ellipticity $e$ of the obtained intensity distribution was measured for different coarsing factors. In all further discussion, the ellipticity calculated from the data while setting the coarsing factor as $k$ is denoted as $e_{k}$. Thus, $e_{k}=S D 2_{k} / S D 1_{k}$. The ellipticity decreased steadily with an increase in the coarsing factor, until it reached a value approaching unity for all speckle sizes. As mentioned above, this is the point where the distribution is seen to be uncorrelated. By further increasing the coarsing factor beyond this point, the ellipticity exhibited small oscillations, but remained approximately equal to 1.0 .

We observed that on steadily increasing the coarsing factor for the calculation of the standard descriptors, the correlation seen in the intensity data was lost when the coarsing factor equals or exceeds the pre-determined minimum speckle size. For example, by examining the case where the minimum speckle size in the simulated speckle pattern was 5 pixels in the data of Fig. 4, it was observed that as long as the coarsing factor was smaller than 5 pixels, the descriptors were able to detect a correlation between the observed intensity values. This results in ellipticity values greater than unity. We refer to this region as the correlated regime. However, when the coarsing factor became larger than 5 pixels, the intensity values detected were seen to be uncorrelated. This resulted in ellipticity values close to unity. We refer to this region as the uncorrelated regime. The minimum speckle size can thus be viewed as the coarsing factor at the transition point between the correlated and uncorrelated regimes.

It is clear from the above discussion that a correlation exists between the minimum speckle size in the speckle pattern and the value of ellipticity at a particular coarsing factor. We observed that for any particular coarsing factor, larger speckle sizes resulted in higher values of ellipticity, unless we are in the uncorrelated regime, in which case $e \approx 1.0$. The characteristic feature of the uncorrelated regime is that the values of ellipticity lie very close to unity and do not continue to decrease monotonically with an increase in coarsing factor. Thus, speckle sizes from multiple speckle fields can be compared by comparing their ellipticity in their intensity distribution, using a low coarsing factor (such as $k=1$, which lies in correlated regime irrespective of the speckle size). 


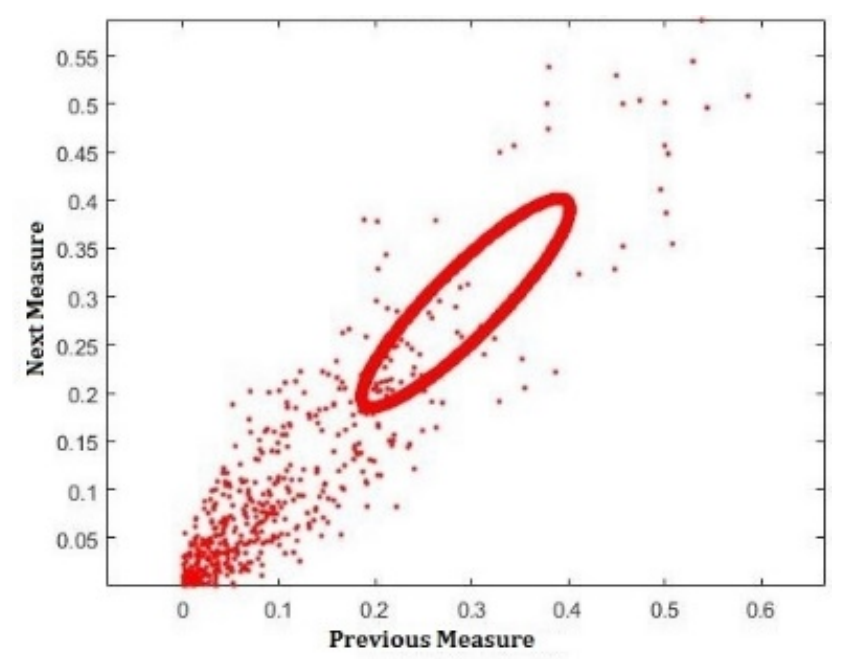

(a)

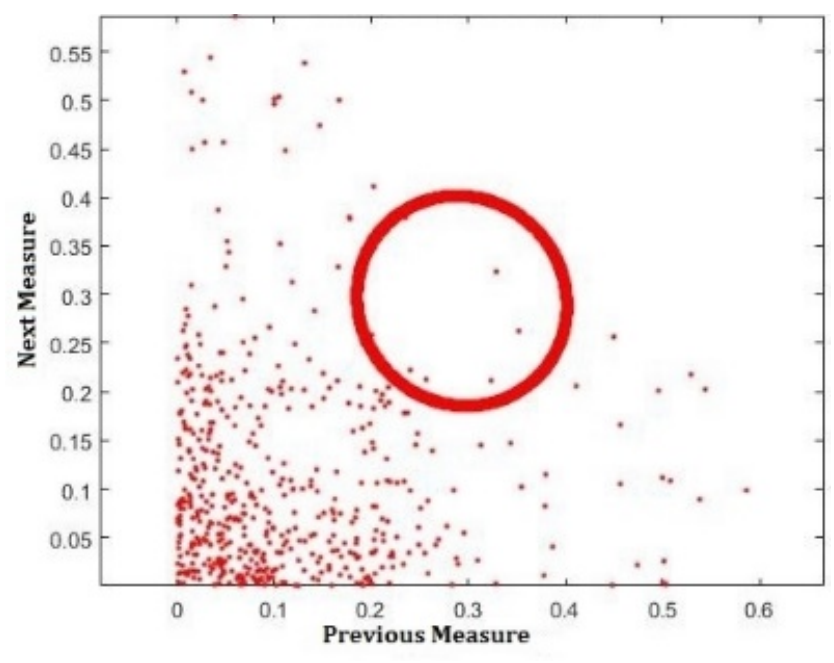

(b)

Fig. 3 Poincaré plot of intensity for minimum speckle size of 5 pixels. (a) When the coarsing factor was 1 pixel, we obtained $S D 1=0.0344$ and $S D 2=0.1490$, giving an ellipticity factor of 4.3314 (b) When the coarsing factor was 6 pixels, we obtained $S D 1=0.1105$ and $S D 2=0.1058$, giving an ellipticity factor of 0.9575 . Thus, the observed correlation is lost at high coarsing factors.

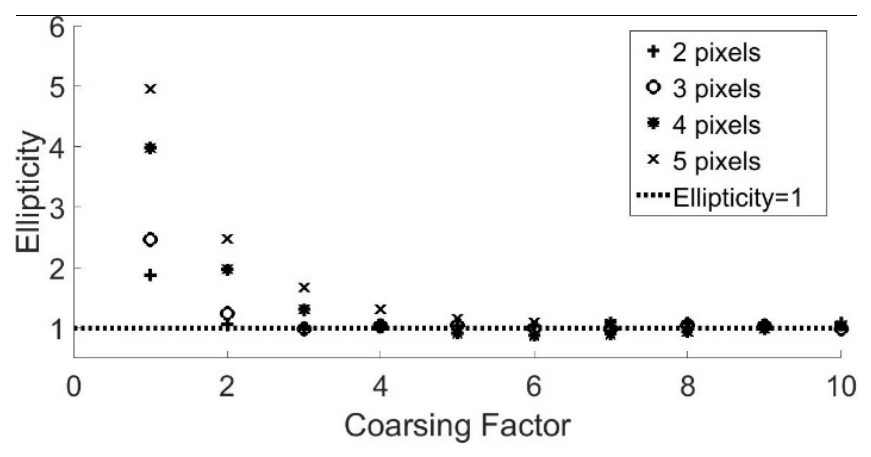

(a)

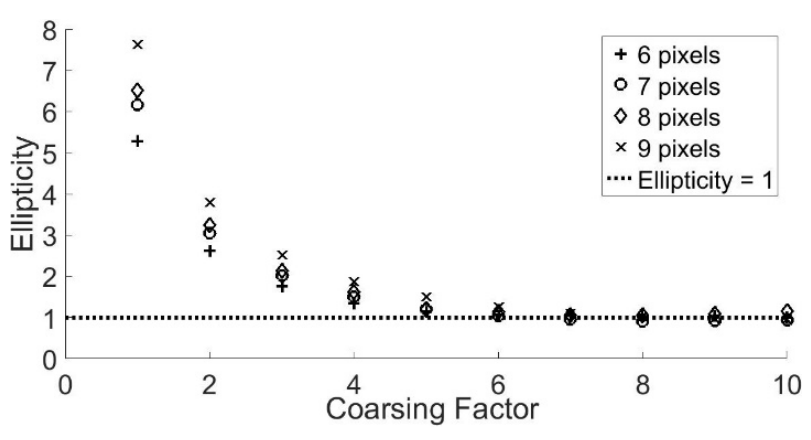

(b)

Fig. 4 Dependence of measured ellipticity on the coarsing factor for minimum speckle sizes of (a) 2 to 5 pixels (b) 6 to 9 pixels. It can be noted that the ellipticity value tends to asymptotically settle around unity, for each of the speckle sizes. We call this asymptotic regime as the uncorrelated regime.

In fact, this ability of the spatial Poincaré plots to distinguish between speckle sizes at low coarsing factors gains significance, especially at larger minimum speckle sizes. Figs. 5(a) and 5(b) demonstrate the challenge with using the PSD for comparing speckle patterns with minimum speckle sizes of 9 and 10 pixels, respectively. According to Eq. (2), the width of the PSD function for the patterns on a $512 \times 512$ window, are roughly 114 and 102 pixels, respectively. This translates to the radius of the functions to be 57 and 51 pixels. This difference of 6 pixels on a field of $512 \times 512$ pixels is more challenging to ascertain, compared to the approach using spatial Poincaré plots, as demonstrated in Fig. 5(c).

For a coarsing factor of $k=1$, which lies in the correlated regime for all speckle sizes greater than 1 pixel, the calculated values of $e_{k}$ for the speckle patterns of 9 and 10 pixels minimum speckle sizes were 7.63 and 8.41 respectively. For $k=2$, these values dropped to 3.80 and 4.18, respectively. Thus, for large speckles, calculating the ellipticity value using a coarsing factor of 1 pixel, for example, provides a method of easier comparison than the visual determination of PSD width.

It is worth recalling that the definition of speckle size has a statistical foundation. It is approximated as the square root of the coherence area of the intensity pattern, which is a function of the covariance of the intensity distribution [9]. As the geometry of this coherence is fundamentally two-dimensional, the process of considering its square root only gives an estimate of the one-dimensional width of the speckle. For small speckles, the variation of pixel intensity in both dimensions is prominent. As such, defining a boundary between which pixels are to be considered "within the speckle" and which are to be considered "outside" is a relatively straightforward task. However, as the size of the speckles increases, the variation in intensity across the pixels becomes more gradual (i.e., the change extends over more pixels). The stark contrast between neighboring pixels diminishes and thus, the 


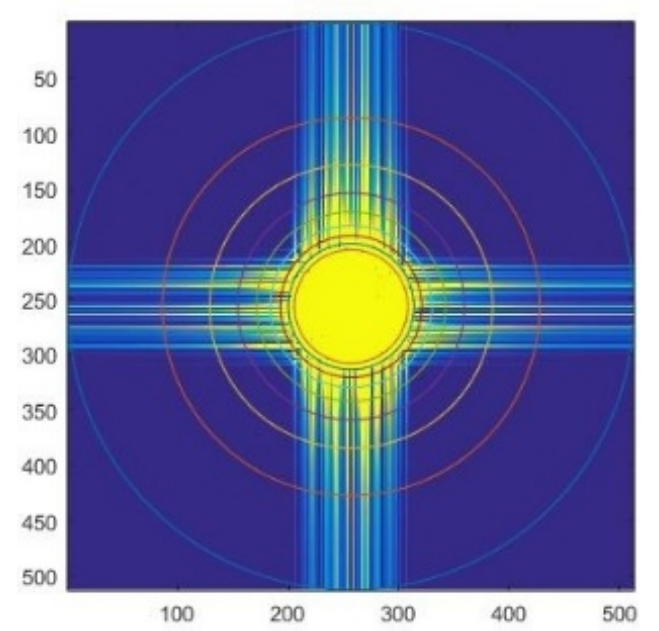

(a)

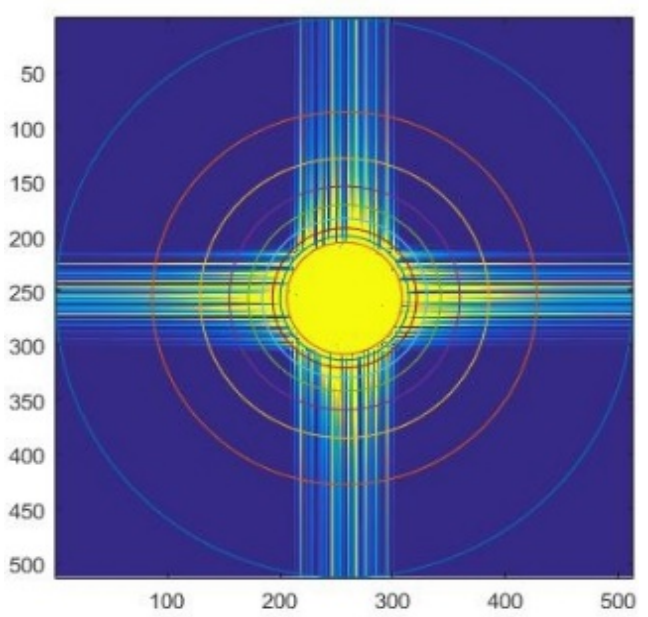

(b)

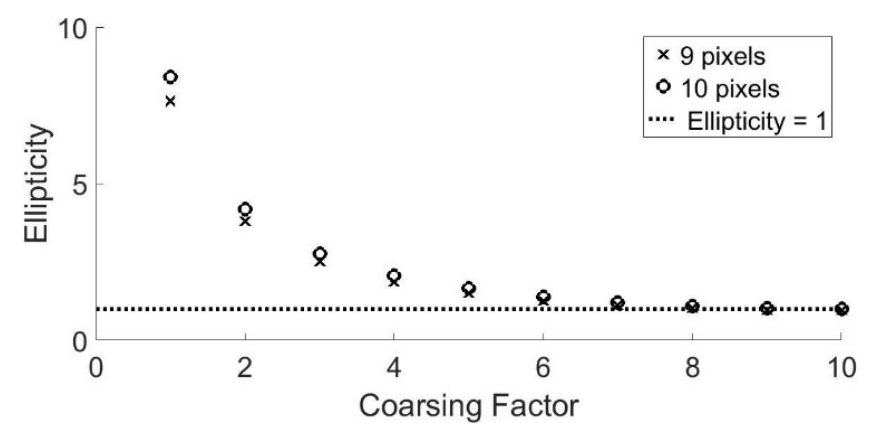

(c)

Fig. 5 Comparison of ease of detecting differences in systems with large minimum speckle sizes: PSD for a minimum speckle size of (a) 9 pixels and (b) 10 pixels compared to the (c) ellipticity values for the same minimum speckle sizes.

boundaries between individual speckles becomes spatially less distinct.

In conclusion, the use of modified Poincaré plot descriptors has been demonstrated as an approach for describing the spatial structure in the measured intensity of a speckle pattern. The Poincaré analysis as presented above, provides a useful approach for estimating the minimum speckle size in a speckle pattern. A comparison was made with the results using an alternative approach, that is, the use of the power spectral density function for the calculation of minimum speckle size. The concept of employing a cutoff ellipticity of $e=1.0$ to identify the minimum sampled speckle size was also introduced. Additionally, it was shown that for large speckles, the present approach has some advantages over the commonly employed approach of assessing the width of the PSD of the speckle pattern. However, at this point the advantages are somewhat qualitative and we have not performed a quantitative assessment of the improvements as this was not the purpose of this study.

Finally, in addition to providing a method for estimating the minimum speckle size in a speckle pattern, spatial Poincaré plots also provide information about the relative contributions of short-term and longterm variations in the spatial structure of the measured intensity distribution of scattered coherent fields.

\section{Disclosures}

The authors declare that there are no conflicts of interest related to this article. 